\title{
REVIEW
}

\section{Cell wall-cytoplasm signalling}

\author{
Augustin Svoboda \\ Department of Biology, Faculty of Medicine, Masaryk University, Brno, Czech Republic
}

Received $30^{\text {th }}$ May 2003.

Revised $27^{\text {th }}$ Januray 2004.

Published online $28^{\text {th }}$ January 2004.

\begin{abstract}
Summary
The cell wall is no longer considered an inert and silent structure on the outside of the cell but instead, seems to be involved in an extensive cross-talk with the cytoplasm. Turgor, osmosensing, mechanical stress and other strains - all are mediated through the wall. The walled cell senses these signals through integral plasma membrane proteins whose extracytoplasmic domains are extended to the wall and intracytoplasmic domains are coupled with the components of cytoplasmic signal pathways. With its dynamic interactions the cell wall is comparable with the extracellular matrix of animal cells. Only a small part of the sensory machinery has been revealed in both plant and fungal cells.
\end{abstract}

Keywords: cross-talk - WAK - cytoskeleton - cell wall integrity - signalling

\section{INTRODUCTION}

The structures covering cell surfaces, i.e., the extracellular matrix in animal cells and the cell wall in prokaryotes, plant and fungi, have been viewed differently. While many functions, such as the involvement in differentiation, cell-to-cell interactions or development of tissues and organs, are ascribed to the extracellular matrix, the cell wall has only been regarded as a mechanical barrier or support for the protoplast taking part in morphogenesis of a cell or plant body. Recent years have brought about considerable progress in our understanding of the cell wall fine structure, regulatory proteins of plasma membranes, i.e., receptors and ligands, and signalling pathways starting at the cell periphery and operating in the cytoplasm. These findings direct our thinking towards a more dynamic view of the plant cell wall, i.e., the structure which is, in fact, an elaborated extracellular matrix (Bolwell 1993, Shibaoka 1994,
Braam 1999, Pilling and Höfte 2003). In this short review I would like to present some recent findings on the intimate relationships of the cell wall and cytoplasm and on the plasma membrane as an interface for a cross-talk between the cell wall and cytoplasm.

\section{CELL WALL STRUCTURE}

The plant cell wall has been described as a complex of carbohydrates and proteins that forms a continuous layer on the cell surface. Current models present the primary cell wall as a mesh of cellulosic microfibrils filled with hemicelluloses, pectins and proteins (Cosgrove 1997, Kohorn 2000). Most of the wall proteins are glycoproteins, structural or enzymatic. Cellulose is a polymer of $\beta-1,4$ linked $\mathrm{D}$-glucose; these polymers are woven together into 
linear bundles of cellulose fibres. Hemicelluloses comprise a family of heteropolymers containing glucose, xylose, arabinose, galactose and fucose. Pectins constitute a group of polygalacturonic acids that vary in side chains with arabinose and galactose (Cosgrove 1997). Both hemicelluloses and pectins intercalate the cellulosic lattice. The cellulose polymers are synthesized in the plasma membrane by enzyme complexes called rosettes (Saxena and Brown 2000, Reiter 2002, Doblin et al. 2002), while hemicelluloses, pectins and enzymes are secreted by endomembrane systems (Cosgrove 1997, Kohorn 2000).

The fungal cell wall, represented here by that of the yeast Saccharomyces cerevisiae, shares $\beta-1,3$ glucan, as a fibrous component, with the plant cell wall, but other polysaccharides and glycoproteins differ markedly. $\beta-1,3$ glucan and mannoproteins constitute the main part of the wall whereas $\beta-1,6$ glucan and chitin are minor components (Lipke and Ovalle 1998, de Nobel et al. 2000). Mannoproteins are localized in the outer layer while fibrous glucan and chitin form the inner part. Recent models show the wall as a latticework made of unit modules, built round a molecule of $\beta-1,3$ glucan (Lipke and Ovalle 1998, Smits et al. 1999).

\section{CELL WALL MODIFICATION}

The wall is plastic in many ways. The yeast cell wall is softened for bud emergence, expands during bud growth, is modified by the addition of bud scars, and becomes more refractory as it ages (Lipke and Ovalle 1998, de Nobel et al. 2000). The wall is remodelled during mating, cell fusion, pseudohyphal formation and spore germination. This remodelling is analogous to wall softening in plant cells during growth and maturation or in response to injury or pathogens (Zandomeni and Schopfer 1994, Schopfer 1996, Kohorn 2000). In protoplasts, it has been found that the cell wall is an important factor for the renewal of morphogenesis (Nečas and Svoboda 1981, Sawabe et al. 1997, Svoboda et al. 2001), for cytokinesis (Gabriel et al. 1994) and pheromone responses (Pokorna and Svoboda 1995). The question arises as to what are the mechanisms inducing metabolic changes in the wall, maintaining cell wall integrity and driving morphogenesis according to the cell's needs. In analogy with the animal extracellular matrix, one could expect that these mechanisms may also be mediated by integral plasma membrane proteins immersed in the cytoplasm and extending into the cell wall. A number of such proteins have recently been reported.

\section{PLASMA MEMBRANE PROTEINS}

Proteins analogous to animal integrin, selectin, cadherin and other adhesion molecules (Schoenwaelder and Burridge 1999) have been sought in plants and, indeed, antibodies raised against human or chicken integrins have been found to bind to the surface of plant cells (Katembe et al. 1997, Swatzell et al. 1999). One of the features of integrins, which is to bind to the RDG motif (Arg-Gly-Asp tripeptide) of fibronectin (Bissell and Nelson 1999), has surprisingly been found in plants (Laval et al. 1999). However, a sequence search of the Arabidopsis genome has revealed no homologues of animal plasma membrane receptors or linkers (Kohorn, 2000). In S. cerevisiae cytoplasmic proteins cross-reacting with integrin antibodies were detected on immunoblots (Holubářová 2002). However, the same antibodies failed to bind to any surface structures in yeast cells, as seen in the fluorescence microscope. The genome databases Blast, Fasta and Proteome did not revealed any $S$. cerevisiae sequence homology with human or chicken integrins (Holubářová 2002).

In $A$. thaliana several plasma membrane proteins have been reported to have a potential signalling role in cytoplasm-cell wall cross-talks. One of them, an arabinogalactan-rich protein (AGP), is highly glycosylated and, with its GPI (glycosyl phosphatidyl inositol) anchor, may connect the plasma membrane with cell wall components (Svetek et al. 1999). Other hot candidates for cell wall-cytoplasmic signaling are WAKs (wallassociated kinases, He et al. 1996, Cosgrove 2001). These serin-threonin kinases are linked to the cell wall, span the plasma membrane and have a cytoplasmic kinaseet domain (Yang 1998). The association of WAKs with the wall is very strong because, during plasmolysis, they remain in the cell wall. WAKs can bind pectin and proteins like GRP (glycin-rich proteins) and seem to be involved in the control of cell expansion and in the response of the plant to injury and pathogen infection (Kohorn, 2001). The coupling of WAKs with cytoplasmic signal pathways has not so far been reported.

In budding yeast, several candidate molecules have been reported; their structure and localization suggest their involvement in cell wall-cytoplasm signaling.

BUD proteins mediate positional information on a new bud (Chant 1999). Three of them are of special importance: bud 10 controlling an axial budding in haploids, and bud 8 and bud 9 having a specific effect on bipolar budding in diploids (Zahner et al. 1996).

BUD 10 is a single-pass transmembrane protein with a 500-amino acid extracellular domain and a 300 amino acid intracellular domain. The 
extracellular domain is highly glycosylated and may serve to anchor to proteins in the cell wall (Roemer et al. 1996). Perturbation of the cell wall structure may then induce molecular changes in BUD 10 and, via an interaction with other intramembranous proteins, a positional signal may be transmitted to the cytoplasm. So far no extracellular ligands for BUD 10 have been reported (Chant 1999).

The bud 8 and bud 9 sequences predict transmembrane proteins with a 450-500 amino acid extracellular domain and a short intracellular domain (Chant 1999). It can be expected that the two proteins perform their function first of all in controlling the budding site, but their large extracellular domain extending deep into the wall may be involved in multiple contacts and may thus transmit information from the wall to the cytoplasm.

Some very promising candidate molecules have recently been found during the analysis of a signaling cascade ensuring cell wall integrity (Levin and Errede 1995, Jung and Levin 1999, van Drogen and Peter 2001). The loss of any component of the cascade results in cell lysis defects under conditions when the cell is exposed to environmental stressors, such as elevated temperature or hypo-osmotic shock (Kamada et al. 1995), mating pheromones (Buehrer and Errede 1997) and calcofluor white, a chitin antagonist (Ketela et al. 1999). The mechanism by which information regarding the state of the cell wall is transmitted to the intracellular signaling apparatus remains unknown. Two major cell surface sensors for the activation of cell integrity signalling have been described. One of them, Wsc1, is responsible for signalling wall stress during vegetative growth and the other one, Mid2, is suggested to signal wall stress during pheromoneinduced morphogenesis (Rajavel et al. 1999). Both Wsc1 and Mid2 are transmembrane proteins that reside in the plasma membrane (Rajavel et al. 1999, Lodder et al. 1999). They both possess a small intracytoplasmic domain, single transmembrane region and extracellular domain rich in Ser/Thr residues and highly mannosylated (Rajavel et al. 1999). The extracellular domains of Wsc1 and Mid2 may thus act as probes of the extracellular matrix (Rajavel et al. 1999).

\section{CYTOPLASMIC TARGETS INVOLVED IN THE CROSS-TALK}

How are the signals from the wall transmitted to the cytoplasm and what are the cytoplasmic targets of these signals?

In animal cells, structural changes in integrins activate adjacent plasma membrane proteins that, in turn, nucleate the formation of large complexes containing both cytoskeletal and catalytic signaling proteins (Clark and Brugge 1995). Regulatory proteins, such as those of the Rho family, are believed to play an important role in stabilizing cell adhesion and regulating cell shape, morphology and mobility (Schoenwaelder and Burridge 1999). The mediating role of actin, as well as that of microtubules and intermediate filaments, has been well established (Schoenwaelder and Burridge 1999).

Both Wsc1 and Mid2, sensors of cell wall perturbations in yeast, are thought to be connected with intracellular biochemical pathways. Recently, it has been found that cytoplasmic domains of these proteins activate ROM2 and RHO1 proteins that, in turn, may switch the cell wall integrity pathway (Philip et Levin 2001). This suggests that both these proteins may be involved in monitoring structural changes in the cell wall and transmitting the relevant information to the cytoplasm.

The BUD 8, BUD 9 and BUD 10 plasma membrane proteins, other possible sensors, may activate subcortically located targets such as BUD 1, BUD 2 and FAR 1 proteins (Chant 1999). These may, in turn, activate actin and microtubules and thus induce morphogenetic events during budding. There are a number of other important proteins in subcortical regions possibly involved in the organization of microtubules and actin such as Kar9 (Miller at Rose 1998), ARP (actin-related proteins, Moreau et al. 1996), tropomyosin (Pruyne at al. $1998 \mathrm{a}, \mathrm{b})$ etc. To what extent these proteins are engaged in the transmission of information from the cell wall to the cytoplasm is not known.

In plant cells, no detail information on the direct connection of WAKs sensors with cytoplasmic signal pathways has been reported. However, a lot of work has been done to define the role of microtubules in shaping the cell.

The growth of a plant cell, occurring over the entire cell surface, is characterized by cell extension. The cell wall of a growing cell contains a microfibrillar network with cellulose microfibrils arranged parallel to one another but transverse to the axis of growth (Kropf et al. 1998). The orientation of cellulose microfibrils seems to be controlled by cortical microtubules also arranged transverse to the axis of elongation. It has been suggested that, by spinning out linear cellulose molecules, the cellulose-synthesizing rosettes are propelled across the membrane surface. They are indirectly guided by microtubules attached to the inner face of the plasma membrane (Cosgrove 2000, Reiter 2002).

The drug treatment experiments and an increasing number of mutants in microtubuleassociated proteins and actin-modulated proteins (Kost et al. 1999, Foster et al. 2003) support the view of these cytoskeletal components as important 
for cell wall formation and maintenance of cell wall integrity.

The use of biochemical, cellular, molecular and genetic approaches has greatly improved our understanding of the cell wall as a dynamic cell compartment. Nevertheless, many questions remain open and more work is needed especially in the definition of molecular sensors and their association with cytoplasmic signal pathways that ensure the appropriate response of the cytoplasm to cell wall signals. In this context the exploitation of recent genomic data will be of great importance.

\section{ACKNOWLEDGEMENTS}

This work was supported by grant No. 310/03/1195 from the Grant Agency of the Czech Republic.

Preliminarily published in J. Berger (ed): Advances in Cell Biology. Kopp Publ., České Budějovice, pp. 113-125, 2003.

\section{REFERENCES}

Bissell M.J. and W.J. Nelson: Cell-to-cell contact and extracellular matrix. Integration of form and function: the central role of adhesion molecules. Curr. Opin. Cell Biol. 11: 537-539, 1999.

Braam J.: If walls could talk - Commentary. Curr. Opin. Plant Biol. 2: 521-524, 1999.

Bolwell G.P.: Dynamic aspects of the plant extracellular matrix. Int. Rev. Cytol. 146: 261324, 1993.

Buehrer B.M. and B. Errede: Coordination of the mating and cell integrity mitogen-activated protein kinase pathways in Saccharomyces cerevisiae. Mol. Cell Biol. 17: 6517-6525, 1997.

Chant J.: Cell polarity in yeast. Annu. Rev. Cell Dev. Biol. 15: 365-391, 1999.

Clark E.A. and J.S. Brugge: Integrin and signal transduction pathways: the road taken. Science 268: 233-239, 1995.

Cosgrove D.J.: Assembly and enlargement of the primary cell wall in plants. Annu. Rev. Cell Dev. Biol. 13: 171-201, 1997.

Cosgrove D.J.: Expansive growth of plant cell walls. Plant Physiol. Biochem. 38: 109-124, 2000.

Cosgrove D.J.: Plant cell walls: wall-associated kinases and cell expansion. Curr. Biol. 11: 558559, 2001.

de Nobel H., H. van den Ende, F.M. Klis: Cell wall maintenance in fungi. Trends Microbiol. 8: 344-345, 2000.
Doblin M.S., I. Kurek, D. Jacob-Wilk, D.P. Delmer: Cellulose biosynthesis in plants: from genes to rosettes. Plant Cell Physiol. 43: 1407-1420, 2002.

Foster R., O. Mattsson, J. Mundy: Plants flex their skeletons. Trends Plant Sci. 8: 202-204, 2003.

Gabriel M.: Karyokinesis and septum formation during the regeneration of incomplete cell walls in protoplasts of Schizosaccharomyces japonicus var. versatilis: a time-lapse microcinematographic study. J. Gen. Microbiol. 130: 625-630, 1984.

He Z.H., M. Fujiki, B.D. Kohorn: A cell wall associated, receptor-like protein kinase. J. Biol. Chem. 271: 19789-19793, 1996.

Holubářová A.: Cell wall as an extracellular matrix: Membrane and submemrane skeleton and its role in morphogenesis of yeast (in Czech language). $\mathrm{PhD}$ Thesis,. Masaryk University Brno 2003, $106 \mathrm{pp}$.

Jung U.S. and D.E. Levin: Genome-wide analysis of gene expression regulated by the yeast cell wall integrity signaling pathway. Mol. Microbiol. 34: 1049-1057, 1999.

Kamada Y., U.S. Jung, R. Piotrowski, D.E. Levin: The protein kinase $\mathrm{C}$-activated MAP kinase pathway of Saccharomyces cerevisiae mediates a novel aspect of the heat shock response. Genes Dev. 9: 1559-1571, 1995.

Katembe W.J., L.J. Swatzell, C.A. Makaroff, J.Z. Kiss: Immunolocalization of integrin-like proteins in Arabidopsis and Chara. Physiol. Plant. 99: 7-14, 1997.

Ketela T., R. Green, H. Bussey: Saccharomyces cerevisiae Mid2p is a potential cell wall stress sensor and upstream activator of the PKC1-MPK1 cell integrity pathway. J. Bacteriol. 181: 3330-3340, 1999.

Kohorn B.D.: Plasma membrane-cell wall contacts. Plant Physiol. 124: 31-38, 2000.

Kohorn B.D.: WAKs; cell wall associated kinases. Curr. Opin. Cell Biol. 13: 529-533, 2001.

Kost B., J. Mathur, N.H. Chua: Cytoskeleton in plant development. Curr. Opin. Plant Biol. 2: 462-470, 1999.

Kropf D.L., S.R. Bisgrove, W.E. Hable: Cytoskeletal control of polar growth in plant cells. Curr. Opin. Cell Biol. 10: 117-122, 1998.

Laval V., M. Chabannes, M. Carriere, H. Canut, A. Barre, P. Rouge, R. Pontlezica, J.P. Galaud: A family of Arabidopsis plasma membrane receptors presenting animal beta-integrin domains. BBA - Protein Struct. Mol. Enzym. 1435: 61-70, 1999.

Levin D.E. and B. Errede: The proliferation of MAP kinase signalling pathways in yeast. Curr. Opin. Cell Biol. 7: 197-202, 1995. 
Lipke P.N. and R. Ovalle: Cell wall architecture in yeast: New structure and new challenges. J. Bacteriol. 180: 3735-3740, 1998.

Lodder A.L., T.K. Lee, R. Ballester: Characterization of the wsc1 protein, a putative receptor in the stress response of Saccharomyces cerevisiae. Genetics 152: 1487-1499, 1999.

Miller R.K. and M.D. Rose: Kar9p is a novel cortical protein required for cytoplasmic microtubule orientation in yeast. J. Cell Biol. 140: 377-390, 1995.

Moreau V., A. Madania, R.P. Martin, B. Winsor: The Saccharomyces cerevisiae actin-related protein arp2 is involved in the actin cytoskeleton. J. Cell Biol. 134: 117-132, 1996.

Necas O. and A. Svoboda: Morphogenesis in protoplasts. In W.N. Arnold (ed.): Yeast cell envelopes: biochemistry, biophysics and ultrastructure. Boca Raton, CRC Press Inc., Vol. 2. 1981, p. 105-128.

Philip B. and D.E. Levin: Wsc1 and Mid2 are cell surface sensors for cell wall integrity signaling that act through Rom2, a guanine nucleotide exchange factor for Rho1. Mol. Biol. Cell 21: 271-280, 2001.

Pilling E. and H. Höfte: Feedback from the wall. Curr. Opin. Plant Biol. 6611-616, 2003.

Pokorná I. and A. Svoboda: Response of yeast protoplasts to their mating partners. Folia Microbiol. 40: 583-587, 1995.

Pruyne D. and A. Bretscher: Polarization of cell growth in yeast I. Establishment and maintenance of polarity states. J. Cell Sci. 113: 365-375, 2000a.

Pruyne D. and A. Bretscher: Polarization of cell growth in yeast II. The role of the cortical actin cytoskeleton. J. Cell Sci. 113: 571-585, 2000b.

Rajavel M., B. Philip, B.M. Buehrer, B. Errede, D.E. Levin: Mid2 is a putative sensor for cell integrity signaling in Saccharomyces cerevisiae. Mol. Cell Biol. 19: 3969-3976, 1999.

Reiter W.D.: Biosynthesis and properties of the plant cell wall. Curr. Opin. Plant Biol. 5: 536542, 2002.

Roemer T., L.G. Vallier, M. Snyder: Selection of polarized growth sites in yeast. Trends Cell Biol. 6: 434-441, 1996.
Sawabe T., Y. Ezura, H. Yamamoto: Plant regeneration from protoplasts of Laminaria japonica Areschoug (Laminariales, Phaeophyceae) in a continuous-flow culture system. Plant Cell Rep. 17: 109-112, 1997.

Saxena I. and R. Brown Jr.: Cellulose synthases and related enzymes. Curr. Opin. Plant Biol. 3: 523531, 2000.

Schoenwaelder S.M. and K. Burridge: Bidirectional signaling between the cytoskeleton and integrins. Curr. Opin. Cell Biol. 11: 274-286, 1999.

Schopfer P: Hydrogen peroxide-mediated cell-wall stiffening in vitro in maize coleoptiles. Planta 199: 43-49, 1996.

Shibaoka H. and R. Nagai: The plant cytoskeleton. Curr. Opin. Cell Biol. 6: 10-15, 1994.

Smits, G.J., J.C. Kapteyn, H. van den Ende, F.M. Klis: Cell wall dynamics in yeast. Curr. Opin. Microbiol. 2: 348-352, 1999.

Svetek, J., M.P. Yadav, E.A. Nothnagel: Presence of a glycosylphosphatidylinositol lipid anchor on rose arabinogalactan proteins. J. Biol. Chem. 274: 14724-14733, 1999.

Svoboda A., I. Slaninová, A. Holubářová: Cytoskeleton in regenerating protoplasts and restoration of cell polarity in the yeast Saccharomyces cerevisiae. Acta Biol. Hung. 52: 325-333, 2001.

Swatzell L.J., R.E. Edelmann, C.A. Makaroff, J.Z. Kiss: Integrin-like proteins are localized to plasma membrane fractions, not plastids, in Arabidopsis. Plant Cell Physiol. 40: 173-183, 1999.

van Drogen F. and M. Peter: MAP kinase dynamics in yeast. Biol. Cell 93: 63-70, 2001.

Yang Z.B.: Signaling tip growth in plants. Curr. Opin. Plant Biol. 1: 525-530, 1998.

Zahner J.E., H.A. Harkins, J.R. Pringle: Genetic analysis of the bipolar pattern of bud site selection in the yeast Saccharomyces cerevisiae. Mol. Cell. Biol. 16: 1857-1870, 1996.

Zandomeni K. and P. Schopfer: Mechanosensory microtubule reorientation in the epidermis of maize coleoptiles subjected to bending stress. Protoplasma 182: 96-101, 1994.

\section{Address:}

Augustin Svoboda, Department of Biology, Medical Faculty, Masaryk University, Joštova 10, 66243 Brno, Czech Republic; asvoboda@med.muni.cz 